\title{
Abundance trends in the thin and thick disks
}

\author{
Sofia Feltzing \\ Lund Observatory, Box 43, SE-221 00 Lund, Sweden
}

\begin{abstract}
The Milky Way harbours two disks that appear distinct concerning scaleheights, kinematics, and elemental abundance patterns. Recent years have seen a surge of studies of the elemental abundance trends in the disks using high resolution spectroscopy. Here I will review and discuss the currently available data. Special focus will also be put on how we define stars to be members of either disk, and how current models of galaxy formation favour that thick disks are formed from several accreted bodies. The ability for the stellar abundance trends to test such predictions are discussed.
\end{abstract}

\section{Current observational knowledge about the thick disk}

During the last decade there has been an increasing interest in trying to establish the chemical abundance trends for the thick disk in the Milky Way. Some of these have included stars from both the thin and the thick disk. These are the most useful ones as they perform differential studies between the two disks. This means that shortcomings in the abundance analyses are, to first order, canceled. This is especially true if the stars are selected to span a small range in effective temperature and surface gravity. Then any resulting difference, at a given $[\mathrm{Fe} / \mathrm{H}]$, between the two disks may be regarded as real.

Recent important observational studies include the following (sorted according to the stellar samples): Differential studies: Fuhrmann, 1998, 10 and 2004, 11, Chen et al., 2000, 8], Mashonkina et al., 2003, 14, Gratton et al., 2003, 13. Bensby et al., 2003, [3] and 2004, 4, Mishenina et al., 2004, 15] Thick disk only: Prochaska et al., 2000, 18] Thin disk only: Reddy et al., 2003, 20], Allende Prieto et al., 2004, 2]

Although the various studies cited above take different approaches to defining their samples and though some of them are only concerned with one of the disks, there is agreement on the following:

- The thick disk is, at a given $[\mathrm{Fe} / \mathrm{H}]$, more enhanced in the $\alpha$-elements than the thin disk

- The abundance trends in the thin disk is a gentle slope

- The solar neighbourhood thick disk stars that have been studied are all old

Other issues are less clear cut. There is some agreement on the following issues (e.g. 14, 3], and Fig. 11 \& 2).

- The thick disk shows evidence of extensive star formation

- The thick disk shows evidence of pollution from SNeIa 
- The thick disk shows evidence of pollution from AGB stars

In addition to the indication of pollution from SNe Ia (i.e. the "knee") found in the $[\mathrm{Mg} / \mathrm{Fe}]$ and $[\mathrm{O} / \mathrm{Fe}]$ trends,for kinematically selected thick disk samples, there is also evidence that thick disk stars well above the galactic plane show the same trend. In Fig. 2 we show the first results of a study of "in situ" metal-rich thick disk stars. It appears that there might be a downward trend in $[\mathrm{Mg} / \mathrm{Fe}]$ as a function of $[\mathrm{Fe} / \mathrm{H}]$ also well above the thin disk. This must, however, be further established before any firm conclusions can be drawn.

There is no agreement on the following two issues ([13, [1], [3], and [5]):

- The thick disk extends to $[\mathrm{Fe} / \mathrm{H}]=0$

- A true age-gap between the end of star formation in the thick disk and the onset of star formation in the thin disk

- Finally: Age-metallicity relation in the thick disk - this is a very tentative statement and, to our knowledge, there is only one study that claims the possibility of such an age-metallicity relation ([5]). This would suggest an extended period of star formation in the thick disk

\section{Ways to define a thick disk star}

Kinematics Selection is clean, even though not trivial, as the velocities of the thick disk overlaps those of the thin. It is easy to compare selection criteria between different studies.

Position Sufficiently high above the galactic plane the thick disk dominates over both the thin disk and the halo. Thus if we can pick stars $1000-1500$ pc above the plane we have a good chance of picking a thick disk star. But, these stars are faint due to their large distance (typically $\mathrm{V} \sim 16$ ) and hence difficult to study. $[\mathbf{F e} / \mathbf{H}]$ and $[\alpha / \mathbf{F e}]$ The metallicity distribution functions of the various stellar populations present in the solar neighbourhood overlap greatly and hence metallicity on its own is not useful for classifying stars. The enhancements of $\alpha$-elements (e.g. O and $\mathrm{Si}$ ) are sometimes used to identify the various populations. There is currently general agreement that the thick disk is enhanced in these elements relative to the thin disk. This should be regarded as something we are looking for rather than prior knowledge that can be used to select stars for further study.

Age Stellar ages can be derived using isochrones. However, such data can not be used to select stars for studies of the thick disk as this must imply a prior knowledge of the age of the stellar population in this disk. This is knowledge that we seek, but do not have.

Given that we in principle can never select a sample of local thick disk stars that is guaranteed to not contain any intervening thin disk stars, I would argue that we should keep the selection schemes as simple and as transparent as possible. In this sense the simplest and most robust selection is based on the kinematics of the stars. This is also the least model dependent method. Of course, should positions be available, i.e. height over the galactic plane, these could, and should, be used. 

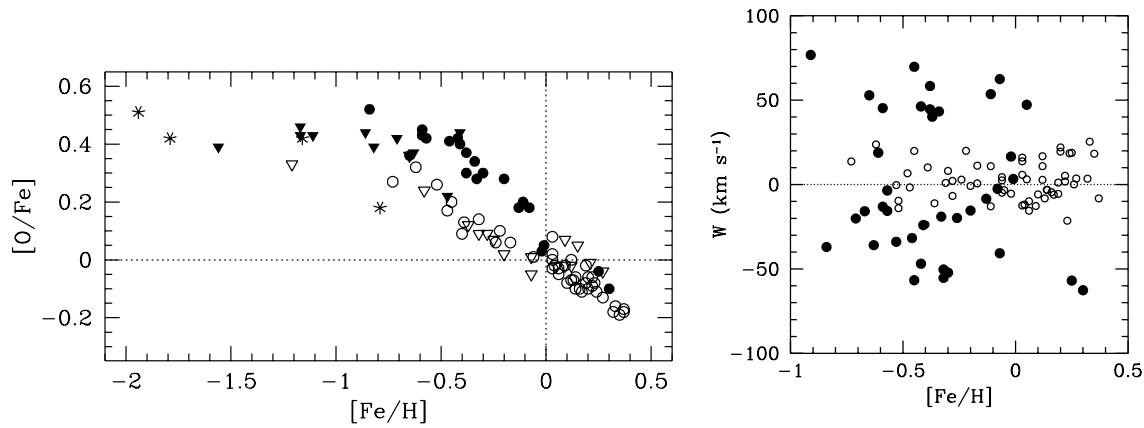

Fig. 1. Left panel: Demonstration that SNeIa have contributed to the chemical enrichment for stars that are kinematically selected to trace the thick disk (i.e. "knee" in the trend for $[\mathrm{O} / \mathrm{Fe}])$. Figure taken from 4. Thick disk stars are represented by filled symbols (circles from [4 and triangles from [16]), thin disk by open symbols (circles from 4 and triangles from [16), and halo stars by * (from [16). Right panel: Shows the $W$-velocity as a function of $[\mathrm{Fe} / \mathrm{H}]$ for stars kinematically selected to be thin (open circles) or thick (filled circles) disk stars. Data taken from 3 and Bensby et al. submitted. This plot establishes that metal-rich stars can also have high velocities perpendicular to the plane (sometimes taken as an evidence for the star to belong to the thick disk)
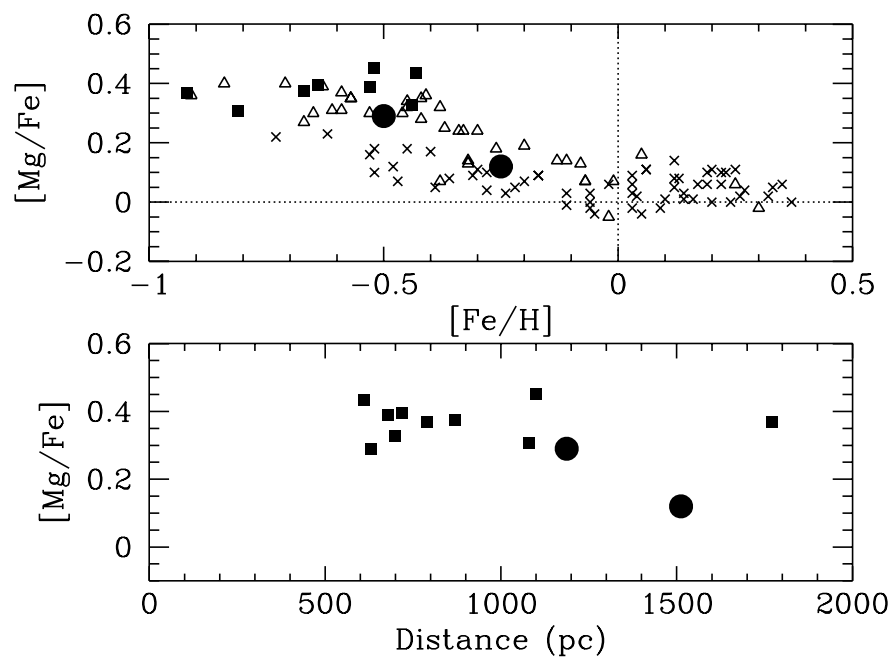

Fig. 2. Upper panel: First results from a study of five "in situ" thick disk stars (Feltzing et al. in prep.). The Mg abundance for two of them are indicated by large • while data from $[\underline{3}$ and Bensby et al. in prep. are shown with $\triangle$ for thick disks stars and $\times$ for thin disk stars. The thick disk stars from [18] are shown as filled squares. Lower panel: $[\mathrm{Mg} / \mathrm{Fe}]$ vs the distance above the galactic plane for the two stars from Feltzing et al. in prep. and the thick disk stars from [18] 


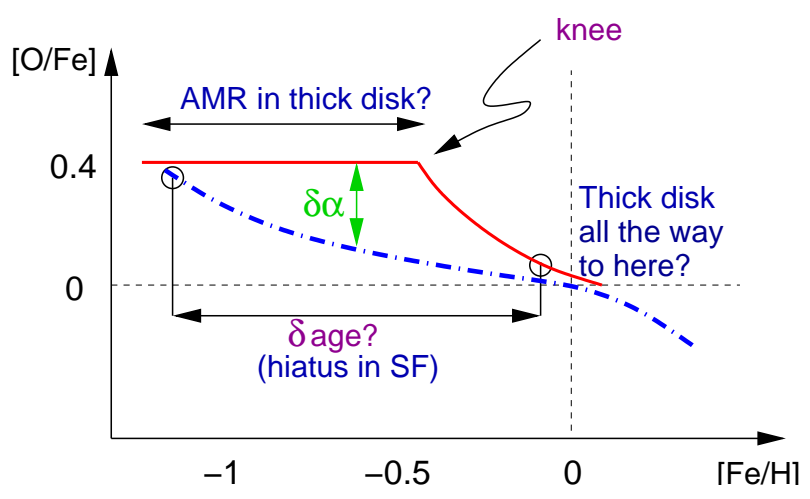

Fig. 3. Summary of current observational knowledge about the thin and the thick disks in the Milky Way. The two trends for $[\mathrm{O} / \mathrm{Fe}]$ vs $[\mathrm{Fe} / \mathrm{H}]$ are depicted in blue, dashed line (thin disk) and red (thick disk). The most debated issues are marked in blue (i.e. thick disk all the way to $[\mathrm{Fe} / \mathrm{H}]=0$, hiatus in star formation, SF, and AMR in thick disk) and topics of some debate in purple ("knee" and $\delta$ age between various sub-populations, such as $\delta$ age between the youngest thin disk and the oldest thick disk). The one issue all agree upon, the $\alpha$-enhancement, is indicated in green (light grey)

\section{Thick disks in other galaxies and how to form them}

\subsection{Thick disks in other galaxies and disks and old stars at high $\mathrm{z}$}

Thick disks are not unique to the Milky Way. Thick disks are seen in many spiral and lenticular galaxies, see e.g. [17, and in galaxies in merging environments, see e.g. 21. Some, 9], even suggest that all spiral galaxies have thick disks. It is an important observational task to verify and extend these findings.

It is also interesting to note that solar metallicities are reported for $z=2$, e.g. 22], and that disks of old stars have been found at redshifts as high as $\mathrm{z}=2.5$, see e.g. 23. These types of findings indicate that indeed the formation of the thick disk in our galaxy might have happened well in the past.

\subsection{Ways to form thick disks}

Meanwhile, the quest to find formation scenarios for the thick disks is ongoing. Earlier studies focused on fast and slow monolithic collapse and various scenarios for increasing the velocity dispersion in a pre-existing thin disk. The latter was envisaged to be able to happen in two distinct ways; either through the general diffusion of orbits and stars being scattered by molecular clouds and other stars or by a violent encounter and merging with a smaller galaxy. A good summary and discussion of why some of these scenarios do not work (e.g. diffusion of orbits and slow collapse) while others (such as fast collapse) is still viable can be found in 12 . Current models of galaxy formation envisages that a galaxy formed from many building blocks. These types of models have only recently become detailed 
enough that we can attempt to compare them in greater detail with the data for the Milky Way, e.g. [1] and [6].

I'll review here three different scenarios that still appear viable (based on the currently available data for the Milky Way, see summary in Fig. 31). For each example I have chosen one or two references that have done detailed models as illustrations to compare the observational data to. This is not an exhaustive account for all the possibilities within each scenario but it gives a flavour of the types of comparisons we ought to make and, hopefully, it also illustrates the shortcomings both of the observed as well as simulated data.

In an early phase with enhanced star formation ([7])

Predictions: no abundance gradients the thick disk; thin disk stars will all have $[\mathrm{Fe} / \mathrm{H}]$ larger than found in the thick disk; period of star formation in thick disk was short $<1$ billion years; thin disk always younger than thick disk

Observations: no vertical gradients; metallicity distributions for the disks overlap; star formation in thick disk includes SNe Ia and AGB; star formation in thick disk probably $>1$ billion years

Conclusions: probably not a viable scenario (if an age-metallicity relation in thick disk is established this formation scenario is in serious trouble)

As the result if violent heating of a pre-existing thin disk ([19])

Predictions: infalling satellite probably has to be large; thin disk younger than thick disk and an age gap between the disks; likely that the abundance trends differ; gradients will be preserved (if they exist in pre-existing thin disk); star formation in original thin disk could be as long as needed (e.g. to create the "knee" in the $\alpha$-element trends)

Observations: no vertical gradients; abundance trends differ; star formation in thick disk includes SNe Ia and AGB

Conclusions: quite possible, no obvious problems with currently available observations

Are made from (largish) satellites that accrete over time ([1], 6]

Predictions: we will only see the abundance trend in the one satellite that finally settles in a torus at our R; the abundance trends will differ depending on the potential well of the satellite, it must be largish and not a dSph; no age gap between thin and thick disk as thin disk is accreted also at early times Observations: tight trends in kinematically selected samples; so far all studied thick disk stars are older than thin disk stars

Conclusions: satellite must be fairly large; only one satellite contributes at the solar radius; the age structure remains a problem (i.e. that very old thin disk stars are predicted and not observed, so far?)

\section{Summary}

There are a number of important observational facts that we now have established for the stellar population of solar neighbourhood stars that have kinematics that are typical of the galactic thick disk. Most importantly it is now convinc- 
ingly shown by independent studies that the abundance trends for kinematically selected samples differ; e.g. the thick disk is more enhanced in $\alpha$-elements than the thin disk. This is also true for other elements as well, e.g. Eu and Ba. From the, currently few, studies of the $\mathrm{r}$ - and the s-process elements it is clear that the thick disk has experienced a contribution from AGB stars.

Some studies find that the thick disk has also experienced a contribution from SNe Ia. This is true in purely kinematically defined samples (e.g. [3]), but is not present, obviously, if the more metal-rich stars, $>-0.5 \mathrm{dex}$, are excluded as in [10.

Based on currently available elemental abundance data and age determinations, the thick disk could have formed either through a violent, heating merger or through accretion of (substantial) satellites in a hierarchical galaxy formation scenario. The fast monolithic-like collapse is getting more and more problematic as data are gathered. It would be especially crucial to establish if there is an age-metallicity relation in the thick disk or not as in that case the thick disk could not have formed in that way (since the models indicate that the formation time-scale for the stars in the thick disk would be very short, see [7]).

Thick disks are common in other galaxies, especially in merger environments, hence perhaps we should prefer the merger scenario for the Milky Way?

\section{References}

1. M.G. Abadi, J.F. Navarro, M. Steinmetz, V.R. Eke: ApJ 597, 21 (2003)

2. C. Allende Prieto, P.S. Barklem, D.L. Lambert, K. Cunha: A\&A 420, 183 (2004)

3. T. Bensby, S. Feltzing, I. Lundström: A\&A 410, 527 (2003)

4. T. Bensby, S. Feltzing, I. Lundström: A\&A 415, 155 (2004)

5. T. Bensby, S. Feltzing, I. Lundström: A\&A 421, 969 (2004)

6. C.B. Brook, D. Kawata, B.K. Gibson, K.C. Freeman: astro-ph/0405306 (2004)

7. A. Burkert, J.W. Truran, G. Hensler: ApJ 391, 651 (1992)

8. Y.Q. Chen, P.E. Nissen, G. Zhao, H.W. Zhang, T. Benoni: A\&AS 141, 491 (2000)

9. J.J. Dalcanton, R.A. Bernstein: AJ 124,1328 (2002)

10. K. Fuhrmann: A\&A 338, 161 (1998)

11. K. Fuhrmann: AN 325, 3 (2004)

12. G. Gilmore, R.F.G: Wyse, K. Kuijken ARA\&A, 27, 555 (1989)

13. R.G. Gratton, E. Carretta, S. Desidera, S. Lucatello, P. Mazzei, M. Barbieri: A\&A 406, 131 (2003)

14. L. Mashonkina, T. Gehren, C. Travaglio, T. Borkova: A\&A 397, 275 (2003)

15. T.V. Mishenina, C. Soubiran, V.V. Kovtyukh, S.A. Korotin: A\&A 418, 551 (2004)

16. P.E. Nissen, F. Primas, M. Asplund, D.L. Lambert: A\&A, 390, 235 (2002)

17. M. Pohlen, M. Balcells, R. Lütticke, R.-J. Dettmar:. A\&A 422, 465 (2004)

18. J.X. Prochaska, S.Q. Naumov, B.W. Carney, A. McWilliam, A.M. Wolfe: ApJ 120, 2513 (2000)

19. P.J. Quinn, L. Hernquist, D.P. Fullagar: ApJ 403, 74 (1993)

20. B.E. Reddy, J. Tomkin, D.L. Lambert, C. Allende Prieto: MNRAS 340, 304 (2003)

21. U. Schwarzkopf, R.-J. Dettmar: A\&A 361, 451 (2000)

22. A.E. Shapley, D.K. Erb, M. Pettini, C.C. Steidel, K.L. Adelberger: ApJ 612, 108 (2004)

23. A. Stockton, G. Canalizo, T. Maihara: AJ 605, 37 (2004) 